Rev Inv Vet Perú 2014; 25(2): 205-212

doi: http://doi.org/10.15381/rivep.v25i2.8492

\title{
EFECTO DEL NIVEL DE FIBRA DETERGENTE NEUTRA SOBRE EL CONSUMO EN LAALPACA (Vicugna pacos)
}

\author{
Effect of Neutral Detergent Fibre on Feed Intake in the Alpaca \\ (Vicugna pacos)
}

\author{
Julio Paredes G. ${ }^{1,2}$, Felipe San Martín H. ${ }^{1,3}$, Juan Olazábal L. ${ }^{1}$, \\ Miguel Ara G. ${ }^{1}$
}

\section{Resumen}

\begin{abstract}
El objetivo del estudio fue evaluar el consumo de materia seca (CMS) y de fibra detergente neutra (CFDN) en la alpaca en función a los niveles de fibra detergente neutra (FDN) en la dieta, así como estimar el consumo potencial de FDN. Se utilizaron cuatro alpacas machos Huacaya de tres años de edad y $48.6 \pm 1.3 \mathrm{~kg}$ de peso vivo $(\mathrm{PV})$, en un diseño Cuadrado Latino $4 \times 4$. El alimento fue suministrado ad libitum, a las 08:00 y 16:00 h. Los tratamientos a base de heno de avena en diferentes proporciones de tallos y hojas, picado en trozos de $2 \mathrm{~cm}$ y separado por un tamiz de $0.4 \mathrm{~cm}$ fueron: hojas de heno de avena (T1), $50 \%$ de heno de avena entero y 50\% de hojas de heno de avena (T2), heno de avena entero (T3) y tallos de heno de avena (T4), que corresponde a $58.24,61.38,66.31$ y $70.22 \%$ de FDN, respectivamente. Los resultados muestran que el incremento del nivel de FDN en la dieta causa disminución del CMS $(\mathrm{p}<0.05)$ sin afectar el CFDN. El CFDN máximo fue de a $0.97 \%$ PV y de 25.6 del peso metabólico (PM). El CFDN promedio fue de $0.92 \pm 0.06 \% \mathrm{PV}$ y $24.22 \pm 1.62 \mathrm{PM}$. Se concluye que el contenido de FDN afecta el consumo de alimento en alpacas en los rangos de FDN evaluados.
\end{abstract}

Palabras clave: camélidos sudamericanos, consumo, fibra detergente neutra

\footnotetext{
${ }^{1}$ Laboratorio de Bioquímica, Nutrición y Alimentación Animal, Facultad de Medicina Veterinaria, Universidad Nacional Mayor de San Marcos, Lima

${ }^{2}$ E-mail: paredesgarcia.j@gmail.com

${ }^{3}$ E-mail:fsanmartinh47@gmail.com

Recibido: 12 de agosto de 2013

Aceptado para publicación: 25 de noviembre de 2013
} 
The objective of this study was to evaluate the dry matter intake (DMI) and neutral detergent fibre intake (NDFI) in the alpaca according to various levels of neutral detergent fibre (NDF) in the diet, and to estimate the intake based on NDF content in the diet. Four 3 -year-old male Huacaya alpacas of $48.6 \pm 1.3 \mathrm{~kg}$ body weight (BW) were used in a $4 \times 4$ Latin Square design. Feed was supplied ad libitum twice daily at 08:00 and 16:00 h. The treatments, based on oat hay with different proportions of stems and leaves, processed by a grinder at the size of $2 \mathrm{~cm}$ and separated by a sieve of $0.4 \mathrm{~cm}$, were: leaves of oat hay (T1), $50 \%$ of oat hay and $50 \%$ of leaves of oat hay (T2), whole oat hay (T3) and stems of oat hay (T4), corresponding to 58.24, 61.38, 66.31 and $70.22 \%$ of NDF respectively. The results showed that increasing the level of NDF in the diet causes a decrease on DMI $(\mathrm{p}<0.05)$ and without affecting NDFI. The maximum NDFI was $0.97 \% \mathrm{BW}$ and $25.6 \mathrm{~g} / \mathrm{kg}^{0.75}$. The average NDFI was $0.92 \pm 0.06 \% \mathrm{BW}$ and $24.22 \pm 1.62 \mathrm{~g} / \mathrm{kg}^{0.75}$. In conclusion, NDF in the range used in the study affects feed intake in the alpaca.

Key words: South American camelids, feed intake, neutral detergent fibre

\section{INTRODUCCIÓN}

Los camélidos sudamericanos (CSA) constituyen una de las actividades productivas y económicas más importantes en la zona alto andina. Su crianza se concentra mayormente en comunidades campesinas, realizándose de manera extensiva, donde la alimentación se basa casi exclusivamente en el pastoreo de la vegetación natural (San Martín, 1996).

La cantidad y calidad nutritiva de las pasturas están influenciadas por una marcada estacionalidad de lluvias. En esas condiciones, la estimación del consumo voluntario de alimento es esencial para formular una dieta que cubra los requerimientos del animal $\mathrm{y}$, en el caso de animales al pastoreo, calcular la disponibilidad del recurso forrajero. De esta manera, el consumo se convierte en uno de los índices más importantes para el buen desempeño productivo del animal (Bustinza, 2001; Chamberlain y Wilkinson, 2002).

Los CSA poseen características digestivas que los diferencian de los rumiantes y que pueden modificar el consumo de alimen- to. Así se tiene, el mayor tiempo de retención del alimento, el pasaje más rápido de agua en el estómago (San Martín, 1987), el mayor volumen de saliva deglutida (Owens e Isaacson, 1977), la mayor frecuencia de ciclos de rumia y la mayor concentración de $\mathrm{NH}_{3}$ en los compartimentos 1 y 2 (Hinderer y von Engelhardt, 1975; von Engelhardt y Schneider, 1977). El consumo de forraje de estos animales, según el NRC (2007), se encuentra entre 1.4 a $2.8 \%$ del peso vivo, en tanto que San Martín (1987) refiere rangos que van de 1.08 a $2.3 \%$ con un promedio de $1.8 \%$ del peso vivo. Estos consumos representan el $74 \%$ de nivel de consumo al pastoreo en comparación al del ovino (San Martín, 1987).

Debido a que el alimento de los CSA es mayormente forraje de baja calidad, la concentración de la fibra detergente neutra (FDN) es el factor que regula predominantemente el consumo, ya que afecta el llenado del estómago, el tiempo de pasaje del alimento y la digestibilidad de la materia seca (Mertens, 2002). Sin embargo, esta relación entre concentración de FDN y consumo de alimento no ha sido suficientemente estudiada en estas especies. Por estas razones, se planteó el presente estudio con el fin de eva- 
Cuadro 1. Análisis químico porcentual del alimento ofrecido en los cuatro tratamientos

\begin{tabular}{lccc}
\hline Tratamientos (heno de avena) & $\mathrm{MS}^{1}$ & $\mathrm{FDN}^{2}$ & $\mathrm{PC}^{3}$ \\
\hline T1 (Hojas) & 91.58 & 58.24 & 6.80 \\
T2(50\% hojas y 50\% entero) & 91.00 & 61.38 & 8.83 \\
T3 (Entero) & 88.51 & 66.31 & 6.36 \\
T4 (Tallo) & 90.72 & 70.22 & 5.75 \\
\hline
\end{tabular}

${ }^{1} \mathrm{MS}=$ Materia seca; ${ }^{2}$ FDN= Fibra detergente neutra, ${ }^{3} \mathrm{PC}=$ Proteína cruda

luar el consumo de materia seca (CMS) y de FDN (CFDN) en función a los niveles de FDN en la dieta en alpaca, así como estimar el consumo potencial de FDN para predecir el CMS, y de esta manera poder mejorar el manejo nutricional.

\section{Materiales y Métodos}

\section{Lugar del Estudio}

El trabajo fue realizado de marzo a junio de 2012 en la Estación Experimental del Centro de Investigación IVITA-Maranganí, localizado a 3727 msnm, en la región Cusco, Perú. Las muestras fueron procesadas en el Laboratorio de Bioquímica, Nutrición y Alimentación Animal de la Facultad de Medicina Veterinaria, Universidad Nacional Mayor de San Marcos, en Lima.

\section{Animales e Instalaciones}

Se utilizaron cuatro alpacas Huacaya machos de 3 años de edad, con peso promedio de $48.6 \pm 1.3 \mathrm{~kg}$. Los animales fueron pesados al inicio del estudio luego de $12 \mathrm{~h}$ de ayuno en una balanza digital de $\pm 100 \mathrm{~g}$ de precisión. Los animales fueron estabulados individualmente en corrales de $1.5 \times 2 \mathrm{~m}$, donde pasaron por una etapa de acostumbramiento de 20 días al consumo del heno de avena (Avena sativa) y se les dosificó contra parásitos internos y externos.
El alimento fue suministrado en cantidad suficiente para obtener un mínimo de $10 \%$ de alimento rechazado. El alimento fue proporcionado a las 08:00 y 16:00 h, recogiendo el alimento de rechazo antes de proporcionar el alimento matutino. El alimento fue pesado en una balanza digital de $\pm 10 \mathrm{~g}$ de precisión.

\section{Tratamientos}

El alimento consistió en heno de avena procesado por una picadora en tamaños de corte de $2 \mathrm{~cm}$. Se utilizaron cuatro tratamientos con diferente contenido de FDN, en base a diferentes proporciones de tallos y hojas.

Los tratamientos fueron:

- T1: Hojas de heno de avena (todo lo que pase por el tamiz de $0.4 \mathrm{~cm}$ )

- T2: $50 \%$ de heno de avena entero y $50 \%$ de hojas de heno de avena

- T3: Heno de avena entero

- T4: Tallos de heno de avena (todo lo que no pase por el tamiz de $0.4 \mathrm{~cm}$ )

Estos tratamientos correspondieron a los niveles de MS, FDN y PC que se señalan en el Cuadro 1. Las cuatro alpacas estuvieron expuestas a los cuatro tratamientos. Cada ensayo tuvo ocho días de adaptación a la dieta y seis días de evaluación, en los cuales se tomaron los datos de consumo diario y de alimento rechazado. 


\section{Alimento Ofrecido y Rechazado}

Se tomaron muestras diarias del alimento ofrecido, haciendo un pool por cada tratamiento. Se determinó el contenido de materia seca (MS) y proteína cruda (PC) de acuerdo a los procedimientos indicados por AOAC (1990), así como el contenido de FDN como describe Van Soest et al. (1994) (Cuadro 1).

Para el alimento rechazado, se tomaron muestras diarias de cada animal por tratamiento y se obtuvo un pool por animal por tratamiento para análisis de MS (AOAC, 1990) y FDN (Van Soest et al., 1994).

\section{Consumo de MS y de FDN}

El CMS y CFDN fue determinado en forma diaria y por animal en cada tratamiento. Para el cálculo, se registró el peso de MS y FDN del alimento ofrecido y rechazado y el consumo se obtuvo de la diferencia entre la MS y FDN del alimento ofrecido y la MS y FDN del alimento rechazado, respectivamente. Los resultados se expresaron en porcentaje de peso vivo y peso metabólico $\left(\mathrm{g} / \mathrm{kg}^{0.75}\right)$.

\section{Análisis de los Datos}

El efecto de los niveles crecientes de FDN (tratamientos) sobre el consumo de MS y de FDN se estimó mediante análisis de varianza para un diseño de cuadrado latinosobrecambio simple (Steel et al., 1997) con cuatro filas (periodos), cuatro columnas (animales) y cuatro tratamientos $(58.24,61.38$, 66.31 y $70.22 \%$ de FDN, respectivamente). La comparación de medias se realizó mediante la prueba de Duncan.

La fuente de variabilidad debida a tratamientos fue descompuesta en un contraste lineal y en una falta de ajuste, con sus respectivas pruebas de hipótesis, de acuerdo a la secuencia sugerida por Kaps y Lamberson (2004). Las funciones de respuesta en consumo de MS y FDN sobre porcentaje de FDN fueron obtenidas mediante análisis de regresión. Los análisis estadísticos fueron realizados con ayuda de los procedimientos PROC GLM y PROC REG del paquete estadístico SAS/STAT ${ }^{\circledR} 9.2$ (SAS Institute Inc., 2009). En todas las pruebas estadísticas se usó un nivel de significancia de 0.05 .

\section{Resultados y Discusión}

El consumo voluntario está determinado por factores que provienen tanto del alimento como del animal (Allen, 1996). Estos factores actúan de manera física o metabólica; sin embargo, por el tipo de dieta habitual de los CSA, se postula que el factor físico es determinante y, por lo tanto, el contenido de la pared celular o FDN del alimento es la fracción que debe tener un rol importante.

Pese a que el estudio se realizó en jaulas individuales y que el forraje fue picado a $2 \mathrm{~cm}$, los animales lograron seleccionar el alimento, dejando como rechazo las partes con mayor contenido de FDN. La alpaca tiene mayor capacidad selectiva entre las partes de la planta que la llama, aunque inferior a la del ovino (San Martín, 1987). Los resultados de ese estudio y los obtenidos por Bryant y Farfán (1984), Huisa (1985) y Reiner y Bryant (1986) permiten señalar que la alpaca es un animal altamente adaptable, debido a su capacidad de selectividad en pastizales nativos.

El CMS varió de 1.38 a $1.73 \%$ del peso vivo (PV) y de 36.34 a 45.67 del peso metabólico (Cuadro 2). Estos valores fueron inferiores a consumos de alfalfa de 1.9-2.4\% PV reportados por Flórez (1973), pero similares a consumos de heno de avena de $1.6 \%$ PV (Fernández Baca y Novoa, 1996) y de $1.8 \%$ PV en una recopilación de información (San Martín, 1987). El CMS del presente estudio es también menor al reportado en otras especies como la oveja (1.41 a $3.9 \%$ PV) y la cabra (1.71 a 5.9\% PV) (NRC, 2007).

El menor consumo es el resultado de factores asociados como el menor requerimiento de energía de los CSA (Schneider et al., 1974), 
Cuadro 2. Consumo de materia seca (MS) en alpacas alimentadas con diferentes niveles de fibra detergente neutra (FDN)

\begin{tabular}{lccc}
\hline Tratamientos (con heno de avena) & $\begin{array}{c}\text { FDN } \\
(\%)\end{array}$ & $\begin{array}{c}\mathrm{PV}^{1} \\
\left(\mathrm{x} \pm \text { d.e. }^{3}\right)\end{array}$ & $\begin{array}{c}\mathrm{PM}^{2} \\
(\mathrm{x} \pm \text { d.e. })\end{array}$ \\
\hline T1 (Hojas) & 58.24 & $1.73 \pm 0.16^{\mathrm{a}}$ & $45.67 \pm 4.03^{\mathrm{a}}$ \\
T2 (50\% hojas y 50\% entero) & 61.38 & $1.65 \pm 0.17^{\mathrm{a}}$ & $43.50 \pm 4.12^{\mathrm{a}}$ \\
T3 (Entero) & 66.31 & $1.38 \pm 0.26^{\mathrm{b}}$ & $36.34 \pm 6.95^{\mathrm{b}}$ \\
T4 (Tallo) & 70.22 & $1.38 \pm 0.26^{\mathrm{b}}$ & $36.36 \pm 6.80^{\mathrm{b}}$ \\
\hline Total & & $1.54 \pm 0.18$ & $40.47 \pm 4.84$ \\
\hline
\end{tabular}

${ }^{1}$ Consumo en base a peso vivo (expresado en porcentaje)

${ }^{2}$ Consumo en base a peso metabólico $\left(\mathrm{g} / \mathrm{kg} P V^{0.75}\right)$

${ }^{3}$ Promedio \pm desviación estándar

a,b Superíndices con letras diferentes dentro de columnas son estadísticamente diferentes entre sí $(p<0.05)$

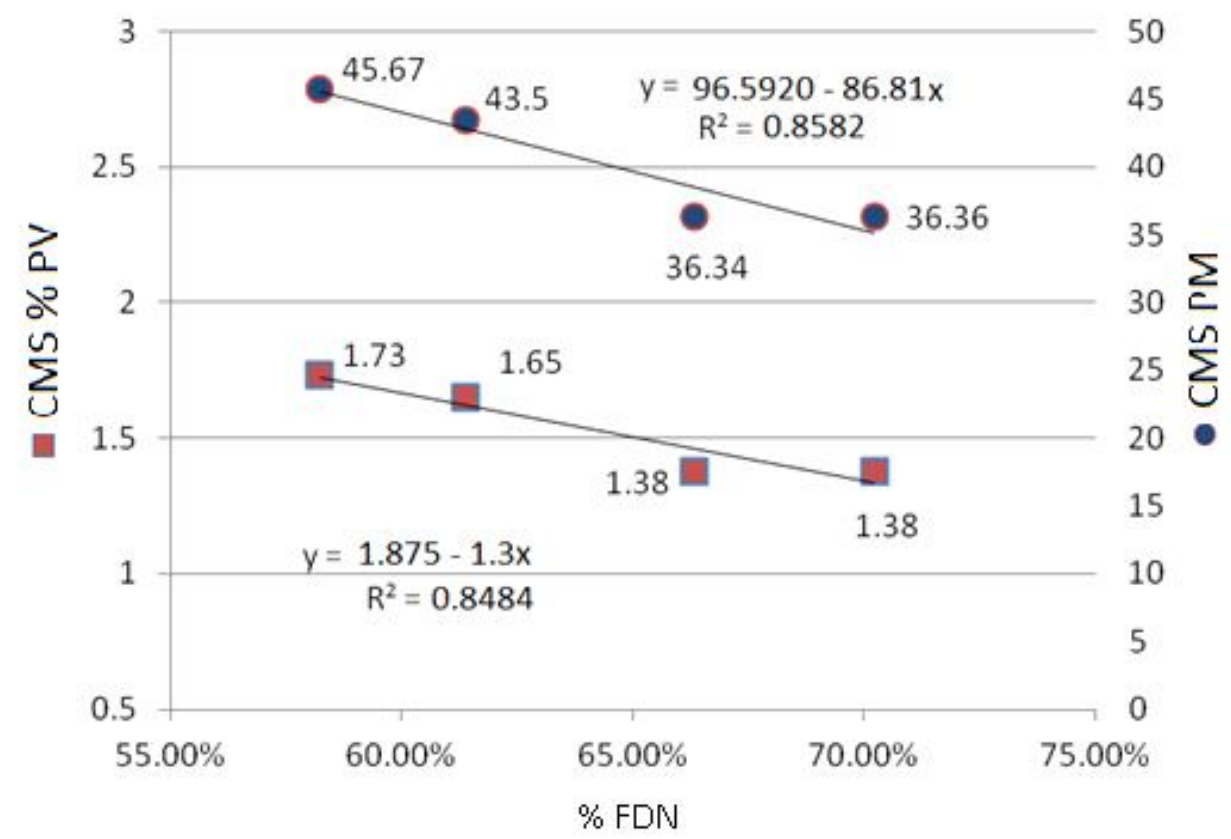

Figura 1. Relación entre CMS (\% Peso vivo [\%PV] y g/kg PV ${ }^{0.75}[\mathrm{PM}]$ ) y contenido de FDN en la dieta (Tratamientos)

el menor volumen de los compartimentos 1 y 2 por unidad de peso metabólico, comparado con el volumen del retículo-rumen en el ovino, y el mayor tiempo de retención del alimento en el tracto digestivo (San Martín, 1987).
Los CMS en T3 y T4 fueron menores que en T1 y T2 (Cuadro 2 ; $\mathrm{p}<0.05$ ). Los resultados evidencian que el componente lineal de la respuesta en CMS a niveles crecientes de FDN fue significativo ( $p<0.05)$, es decir, 
Cuadro 3. Consumo de fibra detergente neutra (FDN) en alpacas alimentadas con diferentes niveles de FDN

\begin{tabular}{lccc}
\hline Tratamientos (con heno de avena) & $\begin{array}{c}\text { FDN } \\
(\%)\end{array}$ & $\begin{array}{c}\mathrm{PV}^{1} \\
\left(\mathrm{x} \pm \text { d.e. }{ }^{3}\right)\end{array}$ & $\begin{array}{c}\mathrm{PM}^{2} \\
(\mathrm{x} \pm \text { d.e. })\end{array}$ \\
\hline T1 (Hojas) & 58.24 & $0.97 \pm 0.13^{\mathrm{a}}$ & $25.6 \pm 3.3^{\mathrm{a}}$ \\
T2 (50\% hojas y 50\% entero) & 61.38 & $0.96 \pm 0.13^{\mathrm{a}}$ & $25.4 \pm 3.3^{\mathrm{a}}$ \\
T3 (Entero) & 66.31 & $0.84 \pm 0.22^{\mathrm{a}}$ & $22.1 \pm 5.9^{\mathrm{a}}$ \\
T4 (Tallo) & 70.22 & $0.90 \pm 0.23^{\mathrm{a}}$ & $23.8 \pm 6.1^{\mathrm{a}}$ \\
\hline Total & $0.92 \pm 0.06$ & $24.2 \pm 1.6$ \\
\hline${ }^{1}$ Consumo en base a peso vivo (expresado en porcentaje) & & \\
${ }^{2}$ Consumo en base a peso metabólico (g/kg PV $\left.{ }^{0.75}\right)$ & & \\
${ }^{3}$ Promedio \pm desviación estándar \\
a,b Superíndices con letras diferentes dentro de columnas son estadísticamente diferentes entre sí \\
$\quad$ (p<0.05)
\end{tabular}

estuvo negativamente afectado por los mayores niveles de FDN en la dieta.

El coeficiente de determinación en la regresión entre el CMS en función al contenido de FDN de los tratamientos fue de 0.8582 (Fig. 1). El CMS fue inversamente proporcional al contenido de fibra de los tratamientos; similar a la información obtenida en llamas por Dulphy et al. (1998), López et al. (2000, 2001), Jalali et al. (2011), en camélidos y otras especies por Meyer et al. (2010), en ovejas por Dulphy et al. (1997) y Aitchison et al. (1986), y en vacunos por Llamas-Lamas y Combs (1991), Dado y Allen (1995) y Arelovich et al. (2008). La relación entre el aumento del contenido de FDN y el descenso del CMS se explica principalmente por el alto volumen de las paredes celulares del forraje (Mertens, 1994), que produce distensión del compartimento estomacal, estimulando los mecano receptores de la capa muscular (Forbes, 1996), limitando el consumo.

El rango de CFDN varió entre 0.84 a 0.97\% PV según el tratamiento (Cuadro 3). Estos valores son inferiores a los rangos obtenidos por Mertens (1987) en bovinos (1.1-
$1.2 \% \mathrm{PV})$, cabras (0.9-1.2\% PV) y ovejas (1.03-1.05\% PV), pero mayor al obtenido en llamas (0.6-0.7\% PV) por Jalali et al. (2011).

En la respuesta del CFDN se observó que no hubo significancia estadística, tanto en la falta de ajuste como en la respuesta lineal, lo que indica que el CFDN en función a los niveles de FDN no fue diferente entre tratamientos. Este resultado se explica por la existencia de un nivel máximo de consumo de FDN a causa del límite potencial de distención estomacal en los niveles usados en este estudio.

A partir de estos resultados se puede deducir que las alpacas consumen la misma cantidad de FDN independientemente del contenido porcentual de FDN de la ración, cuando la concentración de esta fracción del alimento se encuentra entre los niveles de 60 a $70 \%$.

Se puede concluir que el contenido de FDN en el alimento de alpaca es un buen indicador para predecir el consumo y su medición debe ser considerada como relevante (Van Soest, 1994; Meyer et al., 2010). 


\section{Literatura Citada}

1. Aitchison EM, Gil M, Dhanca MS, Osbourn DF. 1986. The effect of digestibility and forage species on the removal of digesta and the voluntary intake of hay by sheep. Br J Nutr 56: 463-476.

2. Allen MS. 1996. Physical constraints on voluntary intake of forages by ruminants. J Anim Sci 74: 3063-3075.

3. $А O A C$. 1990. Official methods of analysis of the Association of Official Analytical Chemists. Vol 1. 15 $5^{\text {th }}$ ed. Washington DC: AOAC. 1298 p.

4. Arelovich HM, Abney CS, Vizcarra A, Galyean ML. 2008. Effects of dietary neutral detergent fiber on intakes of dry matter and net energy by dairy and beef cattle: analysis of published data. Prof Anim Scientist 24: 375-383.

5. Bryant FC, Farfán RD. 1984. Dry season forage selection by alpaca (Lama pacos) in Southern Peru. J Range Manage 37: 330:333

6. Bustinza VC. 2001. La alpaca: crianza, manejo y mejoramiento. Tomo II. Puno: Universidad Nacional del Altiplano. 343 p.

7. Chamberlain A, Wilkinson V. 2002. Consumo voluntario de pienso. En: Alimentación de la vaca lechera. Zaragoza, España: Ed Acribia. p 61-69.

8. Dado RG, Allen MS. 1995. Intake limitations, feeding behavior, and rumen function of cows challenged with rumen fill from dietary fiber on inert bulk. J Dairy Sci 78: 118-133.

9. Dulphy JP, Dardillat C, Jailler M, Jouany JP. 1998. Intake and digestibility of different forages in llamas compared to sheep. Ann Zootech 47: 75-81.

10. Dulphy JP, Martin-Rosset W, Dubroeuc H, Ballet JM, Detour A, Jailler M. 1997. Compared feeding patterns in ad libitum intake of dry forages by horses and sheep. Livest Prod Sci 52: 49-56.

11. Fernández-Baca S, Novoa C. 1996. Estudio comparativo de la digestibilidad de los forrajes en ovino y alpacas. Rev Fac Med Vet UNMSM 18-20: 88-96.

12. Flórez JA. 1973. Velocidad de pasaje de la ingesta y digestibilidad en alpacas y ovinos. Tesis de Bachiller. Lima: Univ Nac Mayor de San Marcos. 46 p.

13. Forbes JM. 1996. Integration of regulatory signals controlling forage intake in ruminants. J Anim Sci 74: 3029-3035.

14. Hinderer S, von Engelhardt W. 1975. Urea metabolism in the llama. J Camp Biochem Physiol 52A: 619-622.

15. Huisa T. 1985. Composición botánica y valor nutricional de la dieta de alpaca (Lama pacos) en la época seca. Tesis de Bachiller. Cusco: Univ San Antonio de Abad del Cusco. $61 \mathrm{p}$.

16. Jalali AR, Norgaard P, Weisbjerg MR, Nielsen MO. 2011. Effect of forage quality on intake, chewing activity, faecal particle size distribution, and digestibility of neutral detergent fibre in sheep, goats, and llamas. Small Ruminant Res 103: 143-151.

17. Kaps M, Lamberson WR. 2004. Biostatistics for animal science. Wallingford: CABI Publishing. $445 \mathrm{p}$.

18. Llamas-Lamas G, Comb DK. 1991. Effect of forage to concentrate ratio and intake level on utilization of early vegetative alfalfa silage by dairy cows. J Dairy Sci 74: 526-536.

19. López A, Morales S, Cabrera R, Arias $M$. 2001. Ingestión y digestibilidad aparente de forrajes por la llama (Lama glama) II. Heno de trébol rosado (Trifolium pratense), heno de ballica (Lolium multiflorum), paja de poroto (Phaseolus vulgaris) y paja de avena (Avena sativa). Arch Med Vet 33: 145152.

20. López A, Morales S, Cabrera R, Urra $X$. 2000. Ingestión y digestibilidad aparente de forrajes por la llama (Lama glama), heno de alfalfa (Medicago sativa) y paja de trigo (Triticum Aestivum) en diferentes proporciones. Arch Med Vet 32: 201-208.

21. Mertens DR. 1987. Predicting intake and digestibility using mathematical- 
models of ruminal function. J Anim Sci 64: 1548-1558.

22. Mertens DR. 1994. Regulation of forage intake. In: Fahey Jr GC (ed). Forage quality, evaluation, and utilization. Madison, WI: Am Soc Agron. p 450-493.

23. Mertens DR. 2002. Physical and chemical characteristics of fiber affecting dairy cow performance. En: Proc Cornell Nutrition Conf. Ithaca, NY. p 125-144.

24. Meyer K, Hummel J, Clauss M. 2010. The relationship between forage cell wall content and voluntary food intake in mammalian herbivores. Mammal Rev 40: 221-245.

25. NRC. 2007. Nutrient requirements of small ruminants. Sheep, goats, cervids and new world camelids. Washington DC: National Academy Press. 18 p.

26. Owens FN, Isaacson HR. 1977. Ruminal microbial yields: factors influencing synthesis and bypass. Fed Proc 36: 198-202.

27. Reiner RJ, Bryant EC. 1986. Botani-cal composition and nutritional quality of alpaca diets in two Andean range land communities. J Range Manage 39: 424-427.
28. San Martín F. 1987. Comparative forage selectivity and nutrition of South American camelids and sheep. Doctoral Thesis. Texas, USA: Texas Tech Univ. $146 \mathrm{p}$.

29. San Martín F. 1996. Nutrición de camélidos sudamericanos y su relación con la reproducción. Rev Argentina Prod Anim 16: 305-312.

30. SAS. 2009. SAS/STAT ${ }^{\circledR} 9.2$ User's Guide. $2^{\text {nd }}$ ed. Cary: The SAS Institute. $870 \mathrm{p}$.

31. Schneider W, Hauffe $R$, von Engelhardt W. 1974. Energy and nitrogen exchange in the llama. Eur Assoc Anim Prod 14: 127-130.

32. Steel RD, Torrie JH, Dickey DA. 1997. Principles and procedures of statistics: A biometrical approach. $3^{\text {rd }} \mathrm{ed}$. New York: McGraw-Hill. 666 p.

33. Van Soest PJ. 1994. Nutritional ecology of the ruminant. $2^{\text {nd }}$ ed. Ithaca, NY: Cornell University Press. 476 p.

34. von Engelhardt W, Schneider W. 1977. Energy and nitrogen metabolism in the llama. Anim Res Develop 5: 68-72. 